\title{
¿Realmente existe convergencia regional en México? Un modelo de datos-panel TAR ${ }^{1}$ no lineal
}

\section{Does regional convergence actually exist in Mexico? A non-linear panel-data TAR model}

\author{
Domingo Rodríguez-Benavides* \\ Miguel Ángel MendozA-GonZÁLEZ** \\ Francisco Venegas-Martínez*
}

\begin{abstract}
This paper analyzes the hypothesis of regional convergence in Mexico for the period of 1970-2012, through a non-linear growth model. The methodology combines three approaches: the panel-data threshold autoregressive (TAR) model, the unit root tests in panel and the computation of the critical values by bootstraping simulation. The empirical results of the nonlinear model applied to the per capita GDP of different groups of states in Mexico suggest that the proposed model is superior to the linear model and show evidence of partial and absolute convergence for the group of the eleven "richer" states in certain sub-periods.
\end{abstract}

Keywords: economic growth; regional convergence in Mexico; non-linear unit root tests in TAR models; panel-data threshold autoregressive (TAR) models.

\section{Resumen}

Este trabajo analiza la hipótesis de convergencia regional en México para el periodo 1970-2012 por medio de un modelo de crecimiento no lineal. La metodología empleada combina tres enfoques: el modelo panel autorregresivo de umbral (TAR, threshold autorregresive), las pruebas de raíces unitarias en panel y el cálculo de los valores críticos a través de simulación bootstraping. Los resultados empíricos del modelo no lineal aplicado al pIB per cápita de distintos grupos de estados de la república mexicana sugieren que el modelo propuesto es superior al modelo lineal y muestran evidencia de convergencia parcial y absoluta para el grupo de las 11 entidades "más ricas" en ciertos subperiodos.

Palabras clave: crecimiento económico, convergencia regional en México, pruebas no lineales de raíces unitarias en panel.

${ }^{1}$ Modelo autorregresivo de umbral (TAR, por sus siglas en inglés).

* Escuela Superior de Economía, IPN, correo-e: domr@economia.unam.mx; fvenegas1111@ yahoo.com.mx

** División de Estudios de Posgrado de la Fac. de Economía, unAM, correo-e: mendozag@unam.mx 


\section{Introducción}

En la mayoría de los estudios sobre el análisis de convergencia regional de México (Juan-Ramón y Rivera-Batiz, 1996; Esquivel, 1999; Carrillo, 2001; Rodríguez-Oreggia, 2002, y Mendoza-González, 2012) se ha encontrado que el crecimiento del PIB per cápita de largo plazo se caracteriza por un proceso de convergencia sigma, desde la década de los cuarenta y hasta mediados de los ochenta, y de divergencia débil, de 1985 en adelante.

Esta evidencia se ha complementado con base en el análisis de convergencia beta, por medio de la aplicación de modelos lineales a submuestras del periodo, considerando como puntos de inflexión 1985 y 1994, con el objetivo de identificar si el GATT o el TLCAN son parte de la explicación de los procesos de divergencia regional en México (véanse al respecto Rodríguez y Sánchez, 2002; Esquivel y Messmacher, 2002; DíazBautista, 2003; Aguayo-Téllez, 2004; Rodríguez-Oreggia, 2005; Chiquiar, 2005; y González-Rivas, 2007).

Asimismo, la discusión sobre convergencia se ha enfocado en demostrar si el crecimiento regional sigue un proceso de convergencia absoluta o condicional, tomando como referencia a la economía nacional o una economía líder regional (Díaz-Pedroza et al., 2009).

Si se toma en cuenta 1970 como el punto de partida, las conclusiones más importantes son que el crecimiento económico regional se caracteriza por un proceso de convergencia condicional en el periodo 1970-2012: convergencia absoluta y condicional de $1970-1985$ y un proceso de divergencia condicional débil de 1985-2012. La estrategia analítica de la mayoría de estos estudios consiste en mostrar que el proceso de convergencia regional termina en 1985 y se modifica hacia uno de divergencia, lo cual implica un rompimiento estructural que puede ser explicado por un cambio de régimen económico, determinado por las nuevas reglas impuestas por GATT o el TLCAN.

Sin embargo, el enfoque metodológico o de modelación que hasta ahora se ha utilizado estudia de manera separada el cambio de régimen económico y su efecto sobre los procesos de convergencia o divergencia. En contraste, este trabajo tiene como objetivo analizar la hipótesis de convergencia regional en México, considerando que los procesos de convergencia y de divergencia regional sigma o beta observados en el periodo 1970-2012 pueden ser parte de un mismo proceso de crecimiento económico; en consecuencia, se tiene que utilizar una metodología que considere que los regímenes de convergencia y divergencia son casos particulares del mismo proceso de crecimiento económico regional.

Desde el punto de vista teórico, Capello (2009) ha identificado una nueva tendencia de planteamientos en los modelos keynesianos del tipo 
Myrdal o Kaldor (Perroux, 1950 y 1955; Myrdal, 1957; y Kaldor, 1970 y 1981), en los cuales originalmente se produce crecimiento económico con divergencia regional (Petrakos, Rodríguez-Pose y Rovolis, 2005); sin embargo, mediante la incorporación de parámetros y nuevas propiedades dinámicas en los procesos, se genera una solución con una ruta de crecimiento constante o de convergencia económica.

También se han desarrollado nuevos argumentos desde la ortodoxia neoclásica para mostrar que la misma teoría puede predecir procesos de divergencia y convergencia económica (Capello, 2009). El mecanismo para lograrlo consiste en introducir economías de escala y de aglomeración en una función de producción, lo cual provoca que el modelo de crecimiento económico simule comportamientos "catastróficos", similares a los modelos de centro-periferia de la nueva geografía económica (Fujita et al., 1999; Baldwin et al., 2003, y Ottaviano y Thisse, 2004) y muy distintos a la predicción de convergencia del neoclásico original (Barro y Sala-i-Martin, 1991, 1992 y 1995; Ramsey, 1928; Solow, 1956; Cass, 1965, y Koopmans, 1965). Incluso, se ha demostrado que la convergencia es una propiedad que se puede derivar no sólo de modelos de crecimiento como el de Solow, sino de aquellos que construyen estados estacionarios cualitativamente diferentes, por ejemplo, el desarrollo y el subdesarrollo (Mayer-Foulkes, 2005, 2009a, 2009b y 2010).

En contraste, en este trabajo se utiliza la especificación de un modelo de crecimiento no lineal propuesto por Beyaert y Camacho (2008), el cual permite examinar, al mismo tiempo, los regímenes de convergencia y divergencia regional para el periodo 1970-2012. La metodología propuesta consiste en un método de estimación que combina tres enfoques: el modelo de umbral, las pruebas de raíces unitarias en panel y el cálculo de los valores críticos a través de simulaciones boostraping. Así, el texto se organiza como sigue: en la primera sección se retoma la literatura empírica sobre la hipótesis de convergencia y divergencia regional en México; en la segunda, se presenta la metodología econométrica del modelo de umbral, las pruebas de raíces unitarias en panel y el cálculo de los valores críticos a través de simulaciones boostraping; mientras que en la última parte se analizan los resultados empíricos de las series del PIB por habitante de las entidades federativas de la república mexicana durante el periodo de estudio. 


\section{Breve revisión de la literatura empírica}

\subsection{Marco teórico y la especificación de la pruebas de convergencia absoluta y condicional}

La hipótesis de convergencia fue desarrollada por Barro y Sala-i-Martin (1991 y 1992; Sala-i-Martin, 1996), como resultado del modelo de crecimiento económico neoclásico para economías cerradas (Solow, 1956; Cass, 1965, y Koopmans, 1965). La hipótesis original establece que el crecimiento de la relación capital-trabajo $(\mathrm{K} / \mathrm{L})$ está inversamente relacionado con su nivel inicial (Galindo y Malgesini, 1994), pero debido a que el ingreso por habitante depende de la relación capital-trabajo, la predicción establece, para economías con niveles de ingreso diferentes inicialmente pero similares en preferencias y tecnologías, que aquellas más pequeñas tienden a crecer a tasas mayores que las más ricas, de forma que en el largo plazo alcanzan el mismo nivel de ingreso de equilibrio.

La implicación más importante es que las desigualdades de ingreso por habitante entre las diferentes economías se reducen hasta desaparecer en el equilibrio de largo plazo. Este planteamiento se conoce como la hipótesis de convergencia absoluta y la especificación original es una función del crecimiento económico de corte transversal en tiempo discreto y lineal, que se soluciona como una ecuación en diferencias de primer orden. Esta hipótesis de convergencia y sus resultados empíricos han sido criticados, ya que el supuesto de homogeneidad en preferencias y tecnologías de las economías resulta poco realista. En su lugar, se ha propuesto el concepto de convergencia condicional, que establece que las economías tienden a reducir sus desigualdades pero no desaparecen en su totalidad, debido a que cada economía tiende a su propio nivel de ingreso de equilibrio de largo plazo. Para ello, los modelos de corte transversal simples se modificaron para incorporar diferenciación económica por medio de variables exógenas (Mankiw et al., 1992) o para considerar economías heterogéneas con preferencias y tecnologías diversas, por medio de los llamados efectos fijos o aleatorios y parámetros individuales para establecer dinámicas al equilibrio para cada economía en una especificación panel lineal (Islam, 1995, 1998).

En la siguiente ecuación se puede resumir toda la discusión teórica y metodológica anterior. En primer lugar, es importante establecer que los niveles de ingreso por habitante están en logaritmo natural $y_{n, t}=\operatorname{In}\left(Y_{n, t}\right)$, por lo que la tasa de crecimiento se define como el diferencial de los ingresos, en logaritmo: $\Delta y_{n, t}=y_{n, t}-y_{n, t-1}$ 


$$
\Delta y_{n, t}=\delta_{n}-\beta_{n,} y_{n, t-1}+\varepsilon_{n, t}
$$

Con las restricciones que se tienen en una especificación de corte transversal, las preferencias y las tecnologías son idénticas $(\delta)$ y la dinámica al equilibrio $(\beta)$ es la misma para cada economía, lo cual implica que, al resolver la ecuación implícita en diferencias para el equilibrio de largo plazo y aplicando esperanzas, el resultado muestra que, en promedio, las economías tiendan al mismo nivel de ingreso de largo plazo $E\left(y_{n}\right)=\delta / \beta$, siempre y cuando la condición de convergencia se cumpla; esto es, que el parámetro $\beta$ sea negativo y en valor absoluto menor que uno.

Por ello, a la hipótesis de convergencia absoluta también se le conoce como convergencia absoluta beta. En una especificación del crecimiento tipo panel, la hipótesis de convergencia es condicional, puesto que ahora el tiempo discreto es importante; las preferencias y las tecnologías son diversas $\left(\delta_{n}\right)$, y las rutas al equilibrio $\left(\beta_{n}\right)$ pueden ser diferentes para cada economía. Por lo tanto, el ingreso de equilibrio de largo plazo es específico para cada una de las economías $E\left(y_{n}\right)=\delta_{n} / \beta_{n}$

La dinámica al equilibrio individual y la convergencia condicional beta se puede cumplir en dos esquemas de hipótesis: si al mismo tiempo se comprueba que las dinámicas individuales de las economías al equilibrio son convergentes a cada uno de sus niveles de equilibrio $|\beta|<1 \forall$ in, y si las dinámicas individuales son iguales entre el grupo de economías y, como tal, se cumple la condición de convergencia en conjunto $\left|\beta_{n}\right|=|\beta|<1$.

Las principales variantes de la ecuación 1 que se han propuesto han consistido en especificar que los ingresos por habitante de las economías siguen un proceso dinámico hacia el equilibrio con respecto a una promedio $g_{n, t}=y_{n, t}-\bar{y}_{t}$; a una economía líder $g_{n, I, t}=y_{n, t}-y_{1, t}$ o, en el marco del análisis de cointegración en panel, si la hipótesis de convergencia es individual o en conjunto. Si se examinan las desigualdades del ingreso y su dinámica con el equilibrio con respecto a la economía promedio, utilizando el método de integración y cointegración en panel, la ecuación 2 es equivalente a la 1 , en el sentido de que se considera la heterogeneidad de las economías. En el marco del análisis de integración y cointegración en panel, ahora las condiciones de convergencia beta se analizan con el parámetro $\rho_{n}$

$$
\Delta g_{n, t}=\delta_{n}+\rho_{n} g_{n, t-1}+\sum_{i=1}^{\rho} \varphi_{n, i} \Delta g_{n, t-i}+\varepsilon_{n, t}
$$

La especificación de la ecuación 2 se construye con el enfoque de una prueba Dickey-Fuller aumentada para el análisis de integración y cointegración; para corregir los sesgos por problemas de autocorrelación serial, 
se incorpora a la especificación la variable endógena rezagada $p$ periodos: $\sum_{n, i}^{\rho} \varphi_{n, t-i}$. Las propuestas metodológicas de estimación econométrica, para probar la diversidad de hipótesis de convergencia beta, han sido resueltas en el análisis de integración en panel por Levin y Lin (1993), actualizada por Levin, Lin y Chu (2001) y por Im, Pesaran y Shin (1997), mientras que para el análisis de cointegración en panel, por Pedroni (1999) y Larsson (2001).

En este esquema general y de menos restricciones analíticas de una especificación de corte transversal de la ecuación de crecimiento lineal, se pueden probar las hipótesis de convergencia beta absoluta y por lo menos dos tipos de convergencia beta condicional. De acuerdo con la ecuación 2, la hipótesis de convergencia beta absoluta entre economías con similares preferencias y tecnologías $\left(\delta_{n}=\delta\right)$ se cumple cuando la tasa de crecimiento del diferencial del ingreso por habitante con respecto a la media es la misma función negativa del diferencial inicial del ingreso por habitante $\left(\left|-\rho_{n}\right|=|-\rho|<1\right)$. Para analizar la convergencia beta condicional, una opción es establecer que las economías son diferentes en preferencias y tecnologías $\left(\delta_{n}=\delta\right)$, lo que se resuelve estimando un modelo panel con efectos fijos o aleatorios y comprobando que la dinámica al equilibrio sea la misma para todas las economías $\left(\left|-\rho_{n}\right|=|-\rho|<1\right)$. El segundo tipo de hipótesis de convergencia beta condicional resulta de revisar si la dinámica al equilibrio es convergente de forma individual para cada economía $(|-\rho|<1 \quad \forall i n)$ y comprobar por tanto que cada economía tiende a su propio nivel de ingreso de equilibrio.

Existe otra forma de concebir la $\beta$-convergencia: se dice que existe $\beta$-convergencia entre regiones si se cumple la relación negativa entre la tasa de crecimiento del ingreso per cápita y el valor inicial del ingreso per cápita, lo cual implica que las regiones más pobres crecen a un ritmo más acelerado que las ricas. En la década de los noventa, diversos estudios se enfocaron sobre la relación entre la tasa de crecimiento del ingreso per cápita y diferentes medidas de estándares de vida en secciones cruzadas, para investigar el proceso de crecimiento.

La hipótesis de convergencia absoluta establece que, si los países convergen, entonces comparten la misma trayectoria en estado estacionario; mientras que la convergencia condicional se refiere a la existencia de rutas paralelas, aunque no precisamente coincidentes. Otra forma de examinar el fenómeno de convergencia regional es la convergencia sigma, la cual consiste en analizar la dispersión del ingreso por persona entre las economías; se dice que este fenómeno se encuentra presente si este indicador tiende a reducirse a través del tiempo. 


\subsection{La literatura empirica sobre el tema}

Los principales estudios que han probado convergencia regional en México (Caraza-Herrasti, 1993; Juan-Ramón y Rivera-Batiz, 1996; DíazPedroza et al., 2009; Esquivel, 1999; Cermeño, 2001; Carrillo, 2001; Díaz-Bautista, 2003, y Mendoza, 2004) coinciden en definir dos grandes periodos, tomando como punto de inflexión 1985, con la finalidad de indagar si, a partir del proceso de liberalización comercial, se ha presentado un proceso de convergencia en comparación con el periodo previo, en el cual la economía mexicana se mantenía prácticamente cerrada.

Con tal fin, estos trabajos emplean el logaritmo natural del PIB por habitante de las 32 entidades federativas, mediante la construcción del indicador de convergencia sigma y la desviación estándar del logaritmo del PIB por habitante. Díaz-Pedroza et al. (2009) sostienen que la hipótesis de convergencia sigma se cumple para el periodo 1970-1985, mientras que prevalece un proceso de divergencia regional para las entidades federativas de la república mexicana en el periodo 1985-2004.

La mayoría de los estudios sobre convergencia regional en México ha sido efectuada bajo el enfoque de convergencia tipo beta, considerando como economía líder al PIв per cápita promedio nacional. Sus resultados se pueden clasificar en dos grandes grupos. El primero tiene que ver con la hipótesis de convergencia absoluta, la cual sostiene que las economías más pobres tienden a crecer a tasas mayores que las de las economías ricas, de modo que a largo plazo tienden al mismo estado estacionario. En el segundo grupo se ubican los resultados que muestran evidencia a favor de la hipótesis de convergencia condicional, cuyo principal postulado es que cada economía tiene su propio estado estacionario y es más bajo el estado estacionario de la economía con la menor tasa de ahorro (la economía pobre).

No obstante, en ambos grupos los años estudiados son importantes para la inferencia. Por ejemplo, Esquivel (1999) y Mendoza (2004) encuentran evidencia que tiende a soportar ambas hipótesis si el periodo analizado empieza en 1940, mientras que, si se considera 1970 como el punto de partida, no es posible soportar la hipótesis de convergencia absoluta pero sí la de convergencia condicional.

Por su parte, Cermeño (2001), por medio de un modelo panel con restricciones en los parámetros, modela la tasa de crecimiento del PIB por habitante de las 32 entidades, con el fin de analizar el proceso de convergencia condicional de 1970-2000. Sus resultados muestran evidencia de convergencia condicional para el total de entidades, así como excluyendo a Campeche y Tabasco (los estados petroleros). 
Mendoza (2004) emplea cuatro modelos de panel con la finalidad de probar la convergencia condicional entre 1970-2002. Comprobó que la especificación más congruente es el modelo de efectos aleatorios, en virtud de que sus parámetros son más estables y muestran evidencia de convergencia condicional en las dos muestras consideradas con todas las entidades y excluyendo Campeche y Tabasco, con tasas de convergencia de 2.6 y $2.5 \%$, respectivamente.

Dentro de los estudios realizados para probar convergencia a la economía líder regional se destaca el de Díaz-Pedroza et al. (2009), quienes efectúan pruebas de raíces unitarias y de cointegración en panel para probar la convergencia de los estados de la de república mexicana con el Distrito Federal, de 1970 a 2004. Mediante la estimación de la versión irrestricta de la prueba (no se establecen restricciones a priori sobre los parámetros) con el método de Mark y Sul (2003), descubren evidencia a favor de la convergencia condicional. Sus estimaciones de la velocidad de convergencia individual indican que ésta es más rápida en las regiones más ricas que en las pobres.

La aparición de bases de datos desagregadas y las peculiares condiciones de las unidades territoriales ha propiciado el surgimiento de diversos estudios empíricos sobre la convergencia regional. Por ejemplo, Cermeño et al. (2009) analizan la dinámica del valor agregado manufacturero per cápita, como proxy del ingreso per cápita de los municipios de México y condados de los Estados Unidos, por medio de un panel dinámico sin regresores exógenos, en el cual consideran el problema del sesgo. Esto demostró que la dinámica del valor agregado per cápita de los condados de Estados Unidos presenta convergencia condicional y poca dispersión de sus estados estacionarios. Por el contrario, en el caso de México, estos autores, encontraron una dinámica congruente con crecimiento estratificado.

En general, los estudios sobre crecimiento regional en México se han enfocado en indagar si se cumple o no la hipótesis de convergencia y de divergencia, como dos aspectos independientes; sin embargo, no consideran la posibilidad de que ambas sean fases del mismo proceso en un modelo de crecimiento regional no-lineal.

Más recientemente, la literatura sobre el crecimiento se ha enfocado en la existencia de clubes de convergencia a nivel de países (Mora, 2005), es decir, de grupos de economías que presentan un patrón homogéneo y convergencia hacia un estado común estable. En este enfoque se emplean diversos métodos de estimación, como los instrumentos de estadística espacial que permiten identificar la dependencia espacial, heterogeneidad espacial y escala espacial, con el fin de detectar la posible presencia de clusters, los cuales pueden depender de la distribución del ingreso per cápita a nivel de regiones (Dallerba, 2005). 


\section{Metodología econométrica y datos}

\subsection{Análisis de la convergencia con modelos autorregresivos panel de umbral (TAR) $)^{1}$}

De acuerdo con Beyaert y Camacho (2008), la metodología propuesta tiene fundamento en la prueba planteada por Evans y Karras (1996), quienes emplean la especificación de la ecuación (2) con el fin de probar la hipótesis de convergencia con datos panel.

Si $\rho_{n}=0$ en (2), entonces las $N$ economías consideradas en la muestra divergen, mientras que, si se cumple $0<-\rho_{n}<1$ para todo $n$, existe convergencia. En tanto, la convergencia es absoluta si $\delta_{n}=0$ para todo $n, \mathrm{y}$, si esto no se cumple, la convergencia es condicional. Se reconoce que el proceso de convergencia no es uniforme; es decir, ciertas economías convergen únicamente si determinadas circunstancias institucionales, políticas o económicas se cumplen; de no ser así, ocasionan divergencia. En otras palabras, puede ser que se cumpla que $0<-\rho_{n}<1$ para todas las economías o regiones consideradas en la muestra bajo determinadas condiciones, pero que $\rho_{n}=0$ sea válido en caso de que éstas no se cumplan. $\mathrm{Al}$ respecto, Beyaert y Camacho (2008) aceptan la posibilidad de que la tasa de convergencia dependa de las condiciones particulares: puede ser que $0<-\rho_{n}<1$ se cumpla para todos las economías en la muestra, pero que su valor específico difiera de acuerdo con las condiciones prevalecientes en el tiempo $(t)$. Según estos autores, un modelo capaz de representar tal comportamiento se puede especificar como:

$$
\begin{aligned}
& \Delta g_{n, t}=\left[\delta_{n}^{I}+\rho_{n}^{I} g_{n, t-1}+\sum_{i=1}^{\rho} \varphi_{n, i}^{I} \Delta g_{n, t-i}\right] I_{\left\{z_{t-1}<\lambda\right\}} \\
& +\left[\delta^{I I}+\rho^{I I}{ }_{n} g_{n, t-1}+\sum_{i=1}^{\rho} \varphi^{I I}{ }_{n, i} \Delta g_{n, t-i}\right] I_{z_{t-1}<\lambda}+\varepsilon_{n, t},
\end{aligned}
$$

donde $I_{\{x\}}$ es una función indicadora que toma el valor de 1 cuando $x$ es verdadero y cero en los otros casos. De esta manera, la dinámica del PIB per cápita puede seguir uno de los dos regímenes posibles en el tiempo $(t)$, los cuales se denominan regímenes I y II, dependiendo de si $z_{t-1}<\lambda \mathrm{o} z_{t-1} \geq \lambda$, respectivamente. Así, el parámetro $\lambda$ representa un "umbral" y la ecuación (3) es propiamente un modelo autorregresivo de umbral (threshold autoregressive, TAR) de la clase introducida primeramente por Tong (1978). A diferencia de la propuesta de Tong, la de Beyaert y Camacho (2008) representa un avance en dos sentidos: primero, ex-

\footnotetext{
${ }^{1}$ La metodología econométrica expuesta en esta sección se basa en Beyaert y Camacho (2008).
} 
tiende el modelo uniecuacional al modelo panel, y, segundo, considera la posibilidad de que los datos no sean estacionarios.

De acuerdo con el planteamiento de Beyaert y Camacho (2008), en el modelo (3) hay divergencia si $\rho_{n}^{I}=\rho^{I I}=0$ para toda $n$; convergencia global si $0<-\rho_{n}^{I}<1$ para todo $n$ e $i=I$, II, y convergencia parcial si $0<$ $-\rho_{n}^{i}<1$, pero $\rho_{n}^{i}=0$ para toda $n$ e $\mathrm{i} \neq \mathrm{j}$. A $z$ en (3) se le conoce como variable de transición, la cual puede ser endógena o exógena. En el procedimiento de estimación de Beyaert y Camacho (2008), se calcula endógenamente y es el enfoque que se sigue aquí. Estos autores proponen estimar la variable de transición, a partir de:

$$
z_{t-}=g_{m, t}-g_{m, t-d}
$$

para algún $m$ y algún $0<d \leq p$, donde $m$ y $d$ no se fijan a priori, sino que también son determinados endógenamente. De esta forma, $z_{i}$ puede ser estacionaria si las economías convergen, todas ellas y para todo régimen, o para algún régimen. Esto quiere decir que la transición de un régimen a otro se relaciona con la tasa de crecimiento de la economía $j$ en los últimos $d$ periodos.

Aunque se puede elegir un $p$ lo suficientemente grande para propiciar que $\varepsilon_{n, t}$ sea ruido blanco para cada $n$, no es posible excluir la posibilidad de correlación contemporánea entre las economías de sección cruzadas del panel. Lo anterior es crucial, ya que, aunque los choques no están serialmente correlacionados, es probable que las economías convergentes se vean afectadas por ellos. Bajo estos supuestos, la matriz de los errores $\varepsilon$ no es diagonal y es muy probable que tenga la siguiente estructura:

$$
V=\Omega \otimes I_{T}
$$

donde $\Omega=\left[\sigma_{n m}\right]_{n, m=1, \ldots, N}$ y $\sigma_{n m}=\operatorname{cov}\left(\varepsilon_{n, t}, \varepsilon_{m, t}\right)$ para todo $t$. Debido a que la estructura de matriz de $\Omega$ es desconocida, el modelo planteado en la ecuación se estima a través del método de mínimos cuadrados generalizados factibles (MCGF). En el proceso de estimación, se impone la restricción $0<\pi_{1} \leq P\left(z_{t-1} \leq \lambda\right) \leq 1-\pi_{1}$, de forma que ningún régimen tiene lugar en menos de la fracción $\pi_{1}$ de la muestra total. Beyaert y Camacho (2008) establecen este $\pi_{1}$ alrededor de 0.10 y 0.15 ; si $\pi_{1}$ cae por debajo de este límite se prefiere el modelo lineal.

Una vez que el modelo de Beyaert y Camacho (2008) se ha estimado, en la ecuación (3), es necesario probar la superioridad de éste con respecto al modelo lineal de Evans y Karrans, planteado en la ecuación (2). Si el modelo no lineal es superior, el siguiente paso es probar convergencia en los coeficientes $\rho$ de (3); si se encuentra evidencia de convergencia, se 
procede a determinar si ésta es absoluta o condicional por medio de los coeficientes $\delta$.

Desde el punto de vista de la linealidad, la hipótesis nula a probar es que el modelo (2) es el apropiado en lugar del modelo alternativo, planteado en (3). El problema aquí es que bajo las pruebas estadísticas convencionales, como la razón de verosimilitud, Wald o las pruebas LM, no siguen la distribución estándar bajo la hipótesis nula, debido a que algunos parámetros, denominados $\lambda, m y d$, no están identificados bajo esta hipótesis, pero sí bajo la alternativa. Con el fin de superar este problema, Beyaert y Camacho (2008) sugieren realizar un procedimiento similar al propuesto por Hansen (1999) y Caner y Hansen (2001) en el modelo TAR uniecuacional, que consiste en obtener los valores críticos a través de simulaciones por bootstrap. El modelo empleado por Beyaert y Camacho (2008) funciona precisamente al extender esta solución al modelo TAR con datos panel. De esta forma, se busca probar la siguiente hipótesis:

$$
H_{0,1}: \delta_{n}^{I}=\delta_{n}^{I I}, \rho_{n}^{I}=\rho_{n}^{I I}, \varphi^{I}{ }_{i, n}=\varphi^{I I}{ }_{i, n},
$$

para todo $n=1, \ldots, N$ y para todo $i=1, \ldots, p$, contra la alternativa de que no todos los coeficientes son iguales en ambos regímenes. Con tal fin, el modelo (2) se estima por mínimos cuadrados generalizados factibles (FGLS, por sus siglas en inglés), y el modelo (3), por el método "grid-FGLs". ${ }^{2}$ Posteriormente, se calcula el valor de la función de verosimilitud en el punto de estimación y obtenemos $L_{12}=-2 \operatorname{In}\left(L_{1} / L_{2}\right)$, donde $L_{1}$ es el valor de verosimilitud del modelo lineal de un régimen, ecuación (1), y $L_{2}$ es el valor de verosimilitud del modelo de dos regímenes, expresado en la ecuación (3). De esta forma, se rechaza la hipótesis nula de linealidad si $L_{12}$ es relativamente grande. Los valores críticos para $L_{12}$ se obtienen de acuerdo con Beyaert y Camacho (2008), en su extensión de la metodología de Caner y Hansen (2001), en la cual emplean el procedimiento bootstraping en el modelo uniecuacional, para permitir la presencia de correlación contemporánea de sección cruzada de los errores descrita en la ecuación (5). En virtud de que no se conoce si las series poseen o no una raíz unitaria, se realizan dos conjuntos de simulación a través de bootstraping. Al primero de ellos se le denomina simulación "bootstrapsin restringir", y se basa en la estimación no restringida del modelo lineal, especificado en (2), mientras que el segundo es el "bootstrap restringido", el cual impone una raíz unitaria limitando $\rho_{\mathrm{n}}=0$ en la ecuación (2). A partir de estas simulaciones, la inferencia acerca de la linealidad se basa

${ }^{2}$ Método de malla, red o enrejado ( $g r i d$ ) de valores, que permite trazar en forma aproximada la función y así obtener el máximo de la misma. 
en el resultado más conservador, es decir, sobre el valor- $p$ más alto del boostraping. Si se rechaza el modelo lineal, el resto del análisis se lleva a cabo sobre el modelo TAR, en la ecuación (3); en caso de que no sea posible rechazarlo, el estudio se lleva a cabo sobre la versión de bootstrap del procedimiento de Evans-Karras propuesto por Beyaert (2006).

Ahora bien, si el modelo (3) es el apropiado, el siguiente paso consiste en probar convergencia contra divergencia con la siguiente hipótesis nula:

$$
H_{0,2}: \rho_{n}^{I}=\rho_{n}^{I I}=0 \forall \text { in }
$$

en la ecuación (3). Si no es posible rechazar la hipótesis planteada en (7), se concluye que hay divergencia en ambos regímenes, en tanto que las hipótesis alternativas de interés que se desprenden de (7) son:

$$
\begin{aligned}
& H_{A, 2}: \rho_{{ }_{n}}^{I}<0, \rho^{I I}<0 \quad \forall i n, \\
& H_{A, 2}: \rho_{n}^{I}<0, \rho_{n}^{I I}=0 \forall i n \\
& H_{A, 2}: \rho_{n}^{I}=0, \rho_{n}^{I I}=0 \forall i n
\end{aligned}
$$

Cuya interpretación es la siguiente: las alternativas (8b) y (8c) implican que la convergencia tiene lugar únicamente bajo el régimen I o el II, respectivamente. En caso de que se rechace la hipótesis nula a favor de alguna de estas dos hipótesis alternativas, Beyaert y Camacho (2008) lo denominan "convergencia parcial". Se debe notar que en el cumplimiento de la hipótesis, nula o alternativa, se supone que los coeficientes $\rho$ satisfacen la misma propiedad para todos las economías en un tiempo específico, lo cual es consistente con la idea de que las series $g_{n, t-}$ del panel son todas $I(0)$ o $I(1)$.

Con el propósito de discriminar entre las tres hipótesis alternativas planteadas en (8), Beyaert y Camacho (2008) sugieren el empleo de varios estadísticos. Uno de ellos es una prueba de tipo Wald para probar la hipótesis alternativa $H_{A, 2 a}$ de convergencia global. En este caso, el estadístico está dado por

$$
R_{2}=t_{I}^{2}+t_{I I}^{2}
$$

donde $t_{I}$ y $t_{I I}$ son estadísticos tipo $t$ asociados con la estimación de $\rho_{n}^{I}$ y $\rho^{I I}{ }_{n}$ respectivamente, en el modelo (3). Si $\hat{\rho}_{n}^{i}$ es el parámetro estimado a través de "grid-FGLs" de $\rho^{i}{ }_{n}$ para cada régimen, entonces el estadístico viene dado por $t_{i}=\hat{\rho}_{n}^{i} / s_{\rho_{n}^{i}}$, para $i=I, I I$. Valores grandes de $R_{2}$ favorecen la hipótesis de convergencia. Ahora bien, para probar la hipótesis de convergencia parcial $H_{A, 2 b}$ se emplea el estadístico $t_{P}$ mientras que para 
probar la hipótesis de convergencia parcial $H_{A, 2 c}$ se utiliza el estadístico $t_{I T}$ Estas dos últimas pruebas son del lado izquierdo. $\operatorname{Si} t_{I}\left(t_{I I}\right)$ es pequeño y $t_{I I}\left(t_{I}\right)$ no, los datos favorecen la hipótesis de convergencia bajo el régimen $I(I I)$ y divergencia bajo los regímenes $I I(I)$. En ambos casos, los valores de probabilidad apropiados se obtienen a través de simulaciones bootstrap. Por último, para concluir el análisis, es necesario discriminar entre convergencia absoluta y condicional. En términos del modelo (3), bajo la hipótesis de que $\rho^{I I}{ }_{n}<0, \forall$ in y $\forall i$, existe convergencia absoluta si $\delta_{n}^{i}=$ $0 \forall$ in y $\forall$ ii. Por el contrario, si el proceso de convergencia toma lugar en sólo uno de los regímenes, por ejemplo en el $I$, entonces habrá convergencia absoluta en dicho régimen si $\delta_{n}^{I}=0 \forall$ in. Al respecto, Beyaert y Camacho (2008) mencionan la posibilidad de que en el modelo de dos regímenes ocurra que haya evidencia de convergencia global, es decir, si $\rho^{i}{ }_{n}<0$ para todo $n$ e $i$, pero $\delta^{i}{ }_{n}=0$ en sólo un régimen. En este caso, se dice que hay convergencia absoluta en un régimen y convergencia condicional en el otro. Los estadísticos propuestos por estos autores se basan en el método de estimación "grid-FGLs" del modelo (3). De manera análoga, los estadísticos propuestos para probar estas hipótesis son extensiones del modelo TAR univariado de Evans y Karrans (1996) para el caso lineal. Los estadísticos $t$ vienen dados por $t\left(\widehat{\delta}_{n}^{i}\right)=\widehat{\delta}_{n}^{i} / s_{\widehat{\delta}_{n}^{i}}$ con $i=I$, II, y $n$ $=1, \ldots, N$, los cuales están asociados con los valores estimados de los términos constantes y están dados por dados por:

$$
\begin{gathered}
\Phi_{a}=\frac{1}{2 N-1}\left\{\sum_{n=1}^{N}\left[t\left(\widehat{\delta}_{n}^{I}\right)\right]^{2}+\sum_{n=1}^{N}\left[t\left(\widehat{\delta}_{n}^{I I}\right)\right]^{2}\right\} \\
\Phi_{b}=\frac{1}{N-1}\left\{\sum_{n=1}^{N}\left[t\left(\widehat{\delta}_{n}^{I}\right)\right]^{2}\right\} \quad \text { y } \Phi_{c}=\frac{1}{N-1}\left\{\sum_{n=1}^{N}\left[t\left(\widehat{\delta}_{n}^{I I}\right)\right]^{2}\right\}
\end{gathered}
$$

Al respecto, Beyaert y Camacho (2008) argumentan que, debido al carácter endógeno de la variable de transición, los valores $p$ del método bootstrap se obtienen de ajustar el modelo lineal a los datos observados. Las reglas de decisión son las siguientes:

Si se rechaza $H_{O, 2}$ en favor de $H_{A, 2 a}$ y además ocurre alguno de los siguientes tres casos:

Si $\Phi_{a}$ es lo suficientemente grande, entonces hay convergencia condicional en ambos regímenes.

Si $\Phi_{b}$ es lo suficientemente grande pero $\Phi_{c}$ no lo es, entonces hay evidencia de convergencia condicional en el régimen I y convergencia absoluta en el régimen II. 
Si $\Phi_{c}$ es lo suficientemente grande y $\Phi_{b}$ no lo es, la convergencia condicional se encuentra presente en el régimen II y la convergencia absoluta tiene lugar en el régimen I

O bien, si $H_{O, 2}$ se rechaza en favor de $H_{A, 2 b}$ y si ocurre que:

$\Phi_{b(c)}$ es lo suficientemente grande, por lo que la convergencia condicional está presente en el régimen I (II).

$\Phi_{b(c)}$ no es lo suficientemente grande, por lo cual la convergencia absoluta ocurre en el régimen I (II).

\section{Resultados}

A continuación se mostrarán los resultados empíricos obtenidos al aplicar la metodología propuesta a los datos de los estados de la república mexicana durante el periodo 1970-2012. Siguiendo la sugerencia de Beyaert y Camacho (2008), se probará convergencia con un subconjunto de regiones o estados que se consideran que a priori puedan converger; progresivamente se añadirán más estados y replicarán las pruebas en forma sucesiva. El primer grupo que se somete a prueba es el de los estados más ricos, en el que fueron incorporados los estados que se encontraron por arriba del promedio nacional, de acuerdo con la información del pIв per cápita del 2010. Como ya es una práctica común en los estudios empíricos que han probado la hipótesis de convergencia en México, se excluyen de este grupo los estados de Campeche y Tabasco, aunque registran altos ingresos, debido a la extracción de petróleo. De esta manera, el primer grupo lo constituyen Aguascalientes, Baja California, Baja California Sur, Coahuila, Chihuahua, Distrito Federal, Nuevo León, Querétaro, Quintana Roo, Sonora y Tamaulipas. Los datos son anuales y provienen de MendozaGonzález (2014); se incorporan en las pruebas en logaritmos naturales. La gráfica 1 presenta el pIв per cápita en logaritmos naturales, para el periodo de estudio de los 11 estados seleccionados dentro del primer grupo. 


\section{Gráfica 1 \\ Logaritmo natural del PIB per cápita anual de los estados más ricos de la república mexicana}

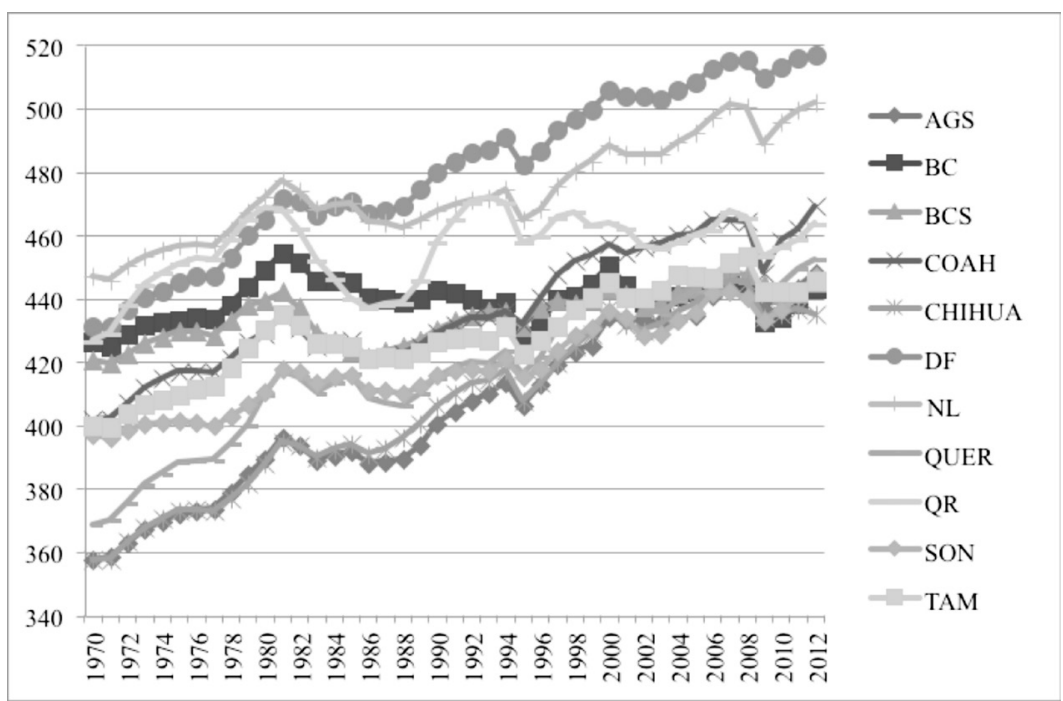

Fuente: elaboración propia con base en Mendoza-González, 2014.

La evolución del PIB per cápita de los estados más ricos muestra una leve tendencia hacia la convergencia, en dos estados: Distrito Federal y Nuevo León que parecen alejarse de la tendencia de largo plazo. Los resultados de las pruebas efectuadas se presentan en el cuadro 1. En el primer panel, $1(a)$, se presentan los resultados de las pruebas del modelo lineal, es decir, de la prueba de Evans y Karras (1996) modificada con bootstraping, ${ }^{3}$ mientras que en el segundo panel, $1(b)$, se encuentran los datos obtenidos de la aplicación del modelo TAR planteado en la ecuación (2). De acuerdo con lo esperado, los resultados del modelo lineal rechazan la hipótesis nula de divergencia con un valor- $p$ de 0.0000 , además de que la prueba revela que esta convergencia ha sido absoluta con un valor- $p$ de 0.9999. En cuanto a los resultados del modelo TAR, las pruebas de linealidad efectuadas rechazan la hipótesis nula de que el modelo lineal es el correcto en virtud de que ambas pruebas, la del irrestricto y la del no restricto, coinciden con este resultado.

\footnotetext{
${ }^{3}$ En todos los casos, el bootstraping se realizó con mil repeticiones.
} 


\section{Cuadro 1 \\ Resultados de las pruebas con los estados más ricos (11) (excluyendo Campeche)}

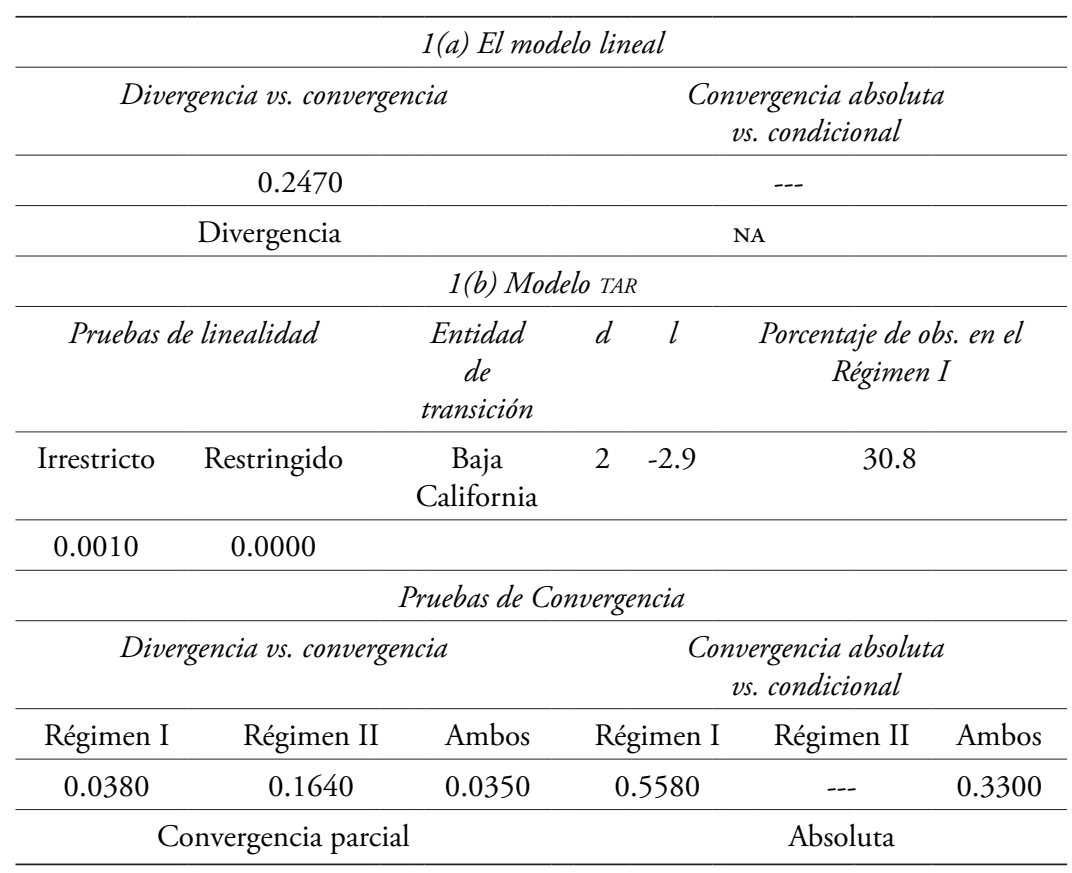

NA: no aplica.

Fuente: elaboración propia.

El estado de Baja California fue elegido endógenamente como la entidad de transición. Interesantemente, esta entidad pasó de la tercera posición en 1970 a la décima en 2010, de acuerdo con la distribución del PIB per cápita por habitante por entidad federativa, lo cual explica en buena medida por qué en el modelo fue elegido como la entidad de transición. El valor estimado del parámetro de rezago es 2 , de forma que la variable de transición es $g_{B C, t}-g_{B C, t-2}$. En lo referente al parámetro de umbral, éste resultó ser - 2.9, lo cual significa que el régimen I corresponde a los ańos en los que la tasa de crecimiento del ingreso per cápita del estado de Baja California fue inferior a la tasa media de crecimiento del grupo en más de 2.9 puntos porcentuales. Es decir, este régimen se refiere a los años en los en que dicha entidad creció en forma más lenta que el resto de los que conforman el grupo de los más ricos. Dicho régimen corresponde a $30.8 \%$ de las observaciones de la muestra, lo cual implica que al régimen II pertenece $69.2 \%$ de las observaciones de la muestra, y se refiere a los años en los que Baja California no crecía en forma tan 
lenta o prosperaba más que la media de este grupo. Los periodos correspondientes a cada régimen y la posición de la variable de transición se muestran en la gráfica 2.

\section{Gráfica 2}

\section{Variable de umbral: Baja California $(d=2)$ para el grupo de los 11 más ricos}

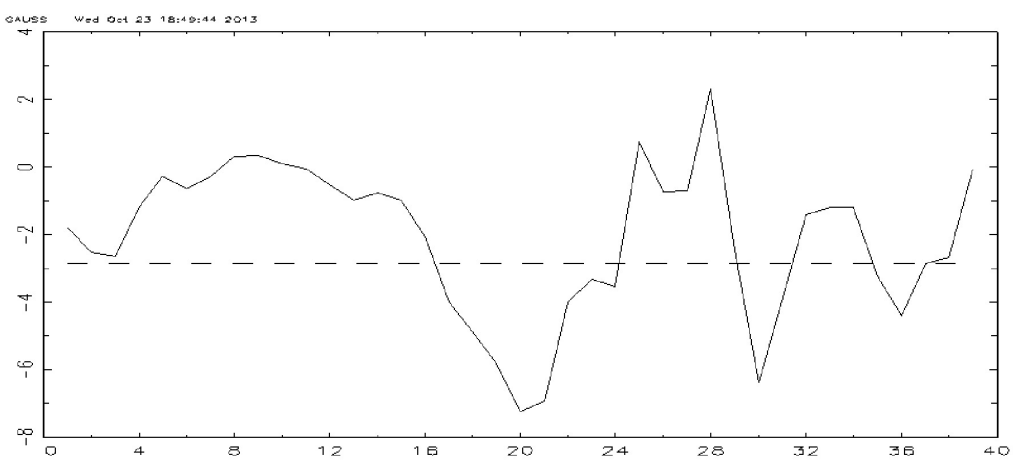

Nota: La línea horizontal se refiere al umbral, mientras que el eje horizontal se refiere al horizonte temporal.

Fuente: elaboración propia.

En la gráfica 2 también se observa un predominio del régimen II en los primeros años de la muestra; es decir, se presenta divergencia a fines de la década de los ochenta, en los años posteriores a la crisis de 1994 que se prolongan hasta el inicio del presente siglo y en los últimos años de la muestra, lo cual sugiere que las reformas llevadas a cabo a finales de los ochenta propiciaron un efecto positivo en lo que al proceso de convergencia se refiere y que las crisis más severas, como las de 1994 y 2008, han incidido negativamente en dicho proceso. En lo que se refiere a la convergencia, la hipótesis nula de divergencia se rechaza únicamente en el régimen I (ver cuadro 1), con un valor- $p$ de 0.0380 ; no obstante, también se rechaza esta hipótesis para ambos regímenes con un valor- $p$ de 0.0350 . Asimismo, las pruebas sugieren que en el régimen I la convergencia es absoluta y más intensiva que bajo ambos regímenes, al presentar valores- $p$ de la prueba de 0.5580 y de 0.3300 , respectivamente. De lo anterior se puede concluir que la convergencia está presente para ambos regímenes en el grupo de los estados más ricos, y se tiene la certeza de que bajo el régimen I la convergencia es absoluta. En consecuencia, estas entidades muestran una trayectoria estable estacionaria únicamente en algunos periodos de la muestra. Luego, se procede a averiguar si el patrón de convergencia de estas 11 entidades se mantiene al incorporar el resto 
de los estados de la república, tomando como referente el promedio de los más ricos, siguiendo el procedimiento de Beyaert y Camacho (2008).

El cuadro 2 muestra los resultados del estudio del proceso de convergencia del promedio de los 11 más ricos combinado con el resto de los estados, es decir, los que se encuentran por debajo del promedio nacional. La gráfica 3 muestra la evolución del logaritmo natural del PIв per cápita para el grupo conformado de esta manera. En la gráfica no se ve alguna tendencia a converger de los miembros de este grupo; por el contrario, se aprecia que el promedio de los 11 más ricos tiende a alejarse del resto. En este caso, los resultados del modelo lineal aplicados a este grupo, mostrados en el cuadro 2(a), indican que no es posible rechazar la hipótesis nula de divergencia, dado que el valor-p para ésta es de 0.3200, y así la prueba consecutiva de convergencia absoluta contra convergencia condicional para el modelo lineal no aplica.

\section{Cuadro 2}

\section{Resultados de las pruebas del promedio de los 11 estados más ricos y el resto que se ubica por debajo del promedio nacional (excluyendo Campeche y Tabasco)}

\begin{tabular}{|c|c|c|c|c|c|}
\hline \multicolumn{6}{|c|}{ 2(a) El modelo lineal } \\
\hline \multicolumn{3}{|c|}{ Divergencia vs. convergencia } & \multicolumn{3}{|c|}{ Convergencia absoluta vs. condicional } \\
\hline \multicolumn{3}{|c|}{0.3200} & \multicolumn{3}{|c|}{---} \\
\hline \multicolumn{3}{|c|}{ Divergencia } & \multicolumn{3}{|c|}{$\mathrm{NA}$} \\
\hline \multicolumn{6}{|c|}{ 2(b) Modelo TAR } \\
\hline \multicolumn{2}{|c|}{ Pruebas de linealidad } & $\begin{array}{l}\text { Entidad de } \\
\text { transición }\end{array}$ & $\lambda$ & \multicolumn{2}{|c|}{$\begin{array}{c}\text { Porcentaje de obs. en el } \\
\text { Régimen I }\end{array}$} \\
\hline Irrestricto & Restringido & Tlaxcala & 1.2 & \multicolumn{2}{|c|}{43.6} \\
\hline 0.0000 & 0.0000 & & & & \\
\hline \multicolumn{6}{|c|}{0.0000} \\
\hline \multicolumn{3}{|c|}{ Divergencia vs. convergencia } & \multicolumn{3}{|c|}{ Convergencia absoluta vs. condicional } \\
\hline Régimen I & Régimen II & Ambos & Régimen I & Régimen II & Ambos \\
\hline \multirow[t]{2}{*}{0.2400} & 0.2340 & 0.1980 & -- & -- & -- \\
\hline & NA & & & NA & \\
\hline
\end{tabular}

NA: no aplica.

Fuente: elaboración propia.

Los resultados del modelo TAR, reportados en el cuadro 2, revelan que el TAR es superior al lineal en ambas pruebas, tanto para el modelo irrestricto como para el restringido. En este caso, la variable de transición resultó ser el de Tlaxcala y el valor estimado del umbral es 1.2. El porcen- 
taje de observaciones en el régimen I es 43.6\%, una fracción superior a la del primer grupo conformado por los 11 más ricos, la cual tiende a prevalecer cuando la diferencia entre la tasa de crecimiento del PIB per cápita de Tlaxcala y el promedio de estos últimos se encuentra por debajo 1.2 puntos porcentuales.

Por el contrario, el régimen II toma lugar cuando la tasa de crecimiento del estado de Tlaxcala se ubica por encima de este nivel. Las pruebas de convergencia aplicadas tanto a cada uno como a ambos revelan que en ningún caso es posible rechazar la hipótesis nula de divergencia. De esta manera, las pruebas efectuadas con el modelo lineal revelaron que no hay ningún tipo de indicio de convergencia entre el promedio de los 11 estados más ricos y el resto de las entidades que se encuentran por debajo del promedio nacional del 2010. Por último, la gráfica 4 muestra la variable de umbral, Tlaxcala $(d=2)$, para el promedio del grupo de los 11 estados más ricos y el resto que se ubica por debajo del promedio nacional (excluyendo Campeche y Tabasco).

\section{Gráfica 3}

Logaritmo natural del PIB per cápita anual del promedio de los 11 estados más ricos y el resto que se ubica por debajo del promedio nacional (excluyendo Campeche y Tabasco)

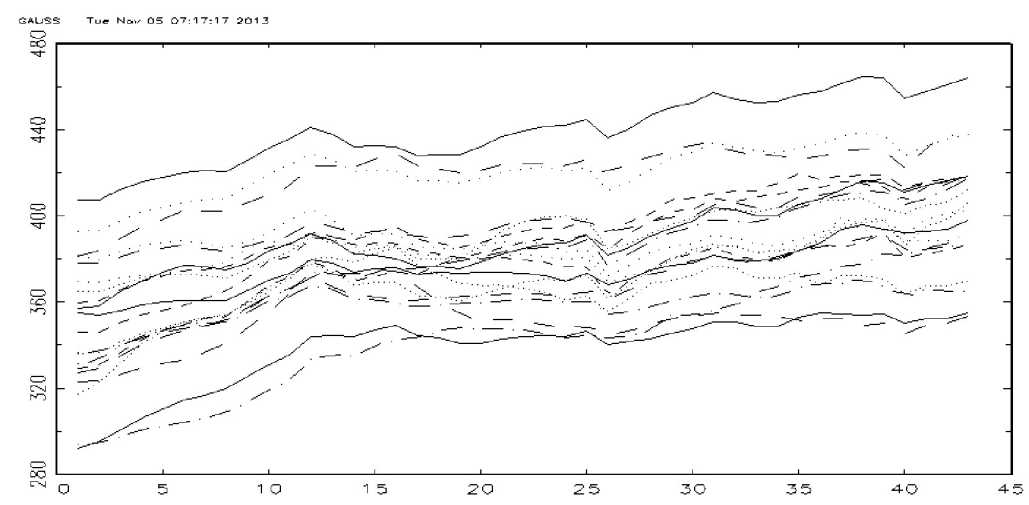

Fuente: elaboración propia. 


\section{Gráfica 4}

Variable de umbral, Tlaxcala $(d=2)$, para el promedio del grupo de los 11 estados más ricos y el resto que se ubica por debajo del promedio nacional (excluyendo Campeche y Tabasco)

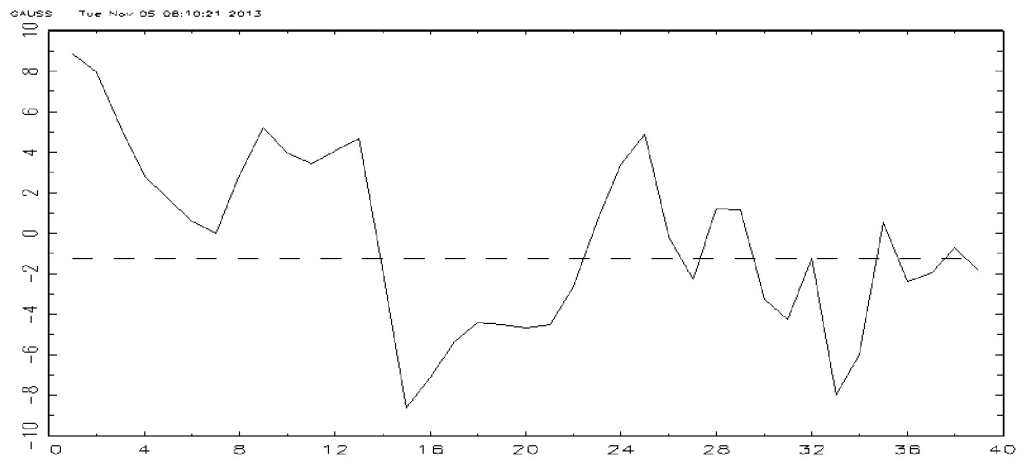

Nota: La línea horizontal se refiere al umbral, mientras que el eje horizontal se refiere al horizonte temporal.

Fuente: elaboración propia.

Por último, como una cuestión interesante, efectuamos el análisis previo a la totalidad de los estados de la república mexicana, con excepción de Campeche y Tabasco, por la razón establecida anteriormente, con la finalidad de averiguar qué información revela el modelo lineal sobre el proceso de convergencia con este nivel de desagregación para México en el periodo de estudio. La evolución del PIB per cápita para este grupo se muestra en la gráfica 5, en la cual se observa un comportamiento similar al del grupo anterior en virtud de que no hay una tendencia clara a converger entre la totalidad de los estados de la republica mexicana; los resultados se presentan en el cuadro 3. De acuerdo con los resultados del modelo lineal aplicados a la totalidad de los estados, al igual que en los casos anteriores, no fue posible rechazar la hipótesis nula de divergencia en el grupo considerado. Sin embargo, a diferencia de los grupos conformados previamente, en este caso las pruebas de linealidad efectuadas sobre el modelo TAR revelan que el modelo lineal es superior al no lineal, por lo que el resto de las pruebas bajo el lineal no aplica para este grupo. De esta manera, los resultados encontrados sobre la totalidad de los estados de México cuestionan los resultados de estudios previos a través de pruebas lineales los cuales sostienen que en nuestro país hay evidencia de convergencia condicional en lugar de convergencia absoluta. Por el contrario, las pruebas efectuadas a través del modelo de Evans y Karras (1996) con bootstraping revelan que, lejos de que se encuentre presente un proceso de convergencia, ya sea absoluta o condicional, hay indicios de di- 
vergencia para la totalidad de los estados en el horizonte de tiempo considerado, lo cual revela que los resultados de la prueba son sensibles a las unidades analizadas en la muestra, de aquí la necesidad de elegir a priori y con un criterio sólido las unidades que se someten a la prueba. Por último, se muestra en la gráfica 6 la variable de umbral, Guanajuato.

\section{Cuadro 3 \\ Resultados de las pruebas de la totalidad de \\ los estados de la república mexicana \\ (excluyendo Campeche y Tabasco)}

\begin{tabular}{|c|c|c|c|c|c|c|}
\hline \multicolumn{7}{|c|}{ 3(a) El modelo lineal } \\
\hline \multicolumn{3}{|c|}{ Divergencia vs. convergencia } & \multicolumn{4}{|c|}{ Convergencia absoluta vs. condicional } \\
\hline \multicolumn{3}{|c|}{0.3330} & \multicolumn{4}{|c|}{--} \\
\hline \multicolumn{3}{|c|}{ Divergencia } & \multicolumn{4}{|c|}{$\mathrm{NA}$} \\
\hline \multicolumn{7}{|c|}{ 3(b) Modelo TAR } \\
\hline \multicolumn{2}{|c|}{ Pruebas de linealidad } & $\begin{array}{l}\text { Entidad de } \\
\text { transición }\end{array}$ & $d$ & $\lambda$ & \multicolumn{2}{|c|}{$\begin{array}{c}\text { Porcentaje de obs. en el } \\
\text { Régimen I }\end{array}$} \\
\hline Irrestricto & $\begin{array}{l}\text { Restrin- } \\
\text { gido }\end{array}$ & Guanajuato & 1 & 0.2 & \multicolumn{2}{|c|}{56.4} \\
\hline 0.6470 & \multirow{2}{*}{\multicolumn{2}{|c|}{ Drunh de comuproncio }} & & & & \\
\hline \multicolumn{6}{|c|}{ Pruebas de convergencia } & \\
\hline \multicolumn{3}{|c|}{ Divergencia vs. convergencia } & \multicolumn{4}{|c|}{ Convergencia absoluta vs. condicional } \\
\hline Régimen I & Régimen II & Ambos & & imen I & Régimen II & Ambos \\
\hline \multirow[t]{2}{*}{---} & -- & --- & & -.- & --- & --- \\
\hline & NA & & & & NA & \\
\hline
\end{tabular}

NA: no aplica.

Fuente: elaboración propia. 


\section{Gráfica 5}

Logaritmo natural del PIB per cápita anual de los estados de las república mexicana: 1970-2012 (excluyendo Campeche y Tabasco)

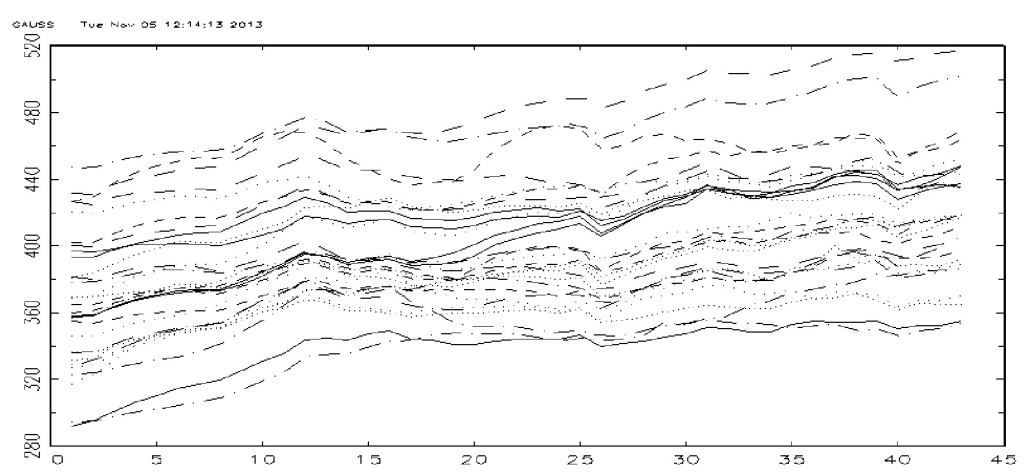

Fuente: elaboración propia.

\section{Gráfica 6}

Variable de umbral: Guanajuato $(d=1)$ para el promedio del grupo de los de los 11 estados más ricos y los primeros 12 estados que se encuentran por debajo del promedio nacional

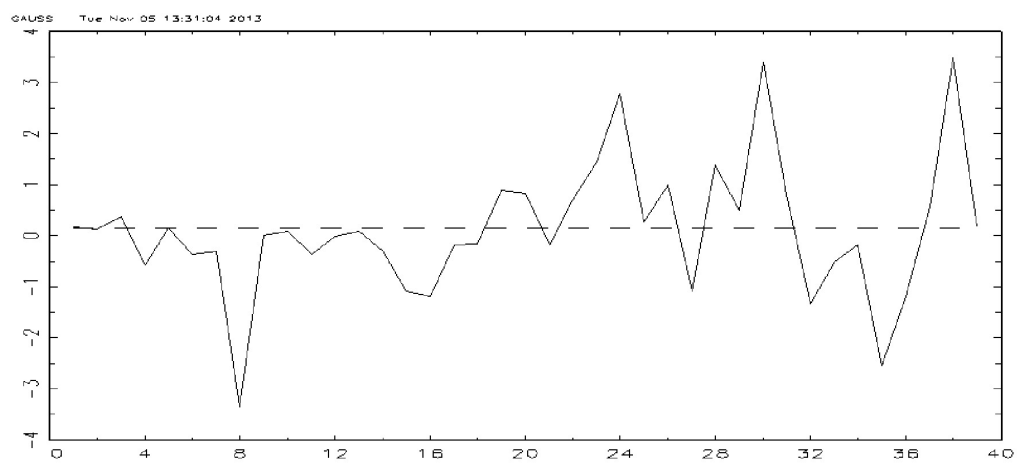

Nota: La línea horizontal se refiere al umbral, mientras que el eje horizontal se refiere al horizonte temporal.

Fuente: elaboración propia.

\section{Conclusiones}

En este trabajo aplicó un modelo no lineal para probar la hipótesis de convergencia en términos del PIB per cápita en la república mexicana. Se contrastaron distintos métodos lineales y no lineales de estimación de datos en panel. A diferencia de los métodos lineales empleados para probar convergencia en el caso de México, el aplicado aquí es una modifica- 
ción del método de Evans y Karras (1996), con simulación bootstraping, lo cual lo hace más robusto. En tanto, el modelo no lineal utilizado pertenece a la clase TAR con dos regímenes, y no sólo permite extender el modelo TAR a los de panel, sino que también añade a la no-linealidad la posibilidad de no estacionariedad atribuible a la presencia de una raíz unitaria en las series del panel considerado. Esta última propiedad es la que hace relevante tal técnica de análisis para probar convergencia o divergencia en un grupo de regiones o países, ya que, si las diferencias del PIB per cápita de un grupo de naciones con respecto a la economía líder - que en este enfoque es el promedio por sección cruzada del gruposon estacionarias, entonces las economías consideradas convergen; de otra manera, divergen si poseen raíces unitarias. $\mathrm{Al}$ aplicar esta metodología al estudio de varios grupos de entidades de la república mexicana, se encontró que las pruebas lineales son incapaces de detectar algún tipo de convergencia en los distintos grupos analizados. El primero lo constituyen los 11 estados más ricos, los cuales se identificaron como aquellos que se encontraron por encima del promedio nacional del 2010. El segundo lo conforman el promedio del primero y el resto de los estados que se ubicaron por debajo del promedio nacional en el 2010. En tanto, el tercer grupo está constituido por la totalidad de los estados de la república mexicana. En todos los grupos considerados se excluyeron Campeche y de Tabasco, por los altos ingresos del componente petrolero.

En conclusión, en el grupo de los 11 más ricos fue posible identificar algunos periodos en los cuales existe convergencia, ya que se vio favorecido por las reformas de primera generación impulsadas a finales de la década de los ochenta y deteriorado en fechas posteriores a las principales crisis en las que se ha visto inmerso México en su historia reciente. Por el contrario, en el segundo grupo no hay indicios de ningún tipo de convergencia en ambos regímenes considerados por el modelo.

Por último, en el caso de las pruebas aplicadas a la totalidad de los estados, los resultados revelaron que el modelo lineal es superior al modelo no lineal. Sin embargo, los datos encontrados por el modelo lineal, al igual que los anteriores grupos, no permitieron rechazar la hipótesis nula de divergencia para este grupo. Esto es relevante porque tiende a cuestionar la evidencia reportada en otros estudios sobre México, que han demostrado la convergencia condicional, ya que, de acuerdo con las pruebas efectuadas a través del modelo de Evans y Karras (1996) modificadas con bootstraping, más bien se encuentra presente un proceso de divergencia cuando se considera la totalidad de los estados. Lo anterior implica que no es conveniente tratar a todos los estados por igual en el análisis empírico, independientemente de la metodología empleada, como generalmente se procede en las pruebas efectuadas con el enfoque esto- 
cástico por medio de pruebas de raíces unitarias y de cointegración en panel en las que no se efectúa algún análisis por grupos, conformados por algún criterio determinado a priori, como en este estudio.

No obstante, la convergencia sí puede estar presente en grupos de estados con características similares y en periodos específicos, lo cual refuerza la idea de que en México también existen clubes de convergencia.

\section{Fuentes consultadas}

Aguayo-Téllez, Ernesto (2004), "Divergencia regional en México, 19902000”, Ensayos. Revista de Economía, XXIII (2), Universidad Autónoma de Nuevo León, Nuevo León, pp. 29-42.

Baldwin, Richard, Rikard Forslid, Philippe Martin, Gianmarco Ottaviano and Frederich Robert-Nicoud (2003), Economic geography and public policy, Princeton University Press, Princeton.

Barro, Robert y X. Sala-i-Martin (1991), "Economic growth in a cross section of countries", The Quartely Journal of Economics, 106 (2), Oxford University Press, Oxford, pp. 407-443.

Barro, Robert y X. Sala-i-Martin (1992), "Convergence", Journal of Political Economy, 100 (2), University of Chicago, Chicago, pp. 223-251.

Barro, Robert Joseph y Xavier Sala-i-Martin (1995), Economic growth, MacGraw-Hill, Nueva York.

Beyaert, Arielle (2006), "Convergence des revenus au sein de l'Union Européenne: une evaluation économétrique”, en H. Capron (ed.), Convergence et dynamique d'innovation au sein de l'espace européen, Editions de Boeck Université, Bruselas, pp. 53-59.

Beyaert, Arielle y Máximo Camacho (2008), "TAR panel unit root tests and real convergence", Review of Development Economics, 12 (3), Social Science Electronic Publishing, pp. 668-681.

Caner, Mehmet y Bruce Hansen (2001), "Threshold autoregression with a unit root", Econometrica, 69 (6), Wiley Online Library, pp. 1555-1596.

Capello, Roberta (2009), “Space, growth and development”, en Roberta Capello y Peter Nijkamp (eds.), Hanbook of regional growth and 
development theories, Edward Elgar Publishing, Gran Bretaña, pp. 33-52.

Caraza-Herrasti, María Inés (1993), "Convergencia del ingreso en la república mexicana”, tesis de licenciatura en Economía, ITAM, México.

Carrillo, Mario (2001), "La teoría neoclásica de la convergencia y la realidad del desarrollo regional en México", Problemas del Desarrollo, 127 (32), Universidad Nacional Autónoma de México, México, pp. 107-134.

Cass, David (1965), "Optimum growth in an aggregative model of capital accumulation: a Turnpike Theorem", The Review of Economic Studies, 32 (3), Oxford University Press, Oxford, pp. 233-240.

Cermeño, Rodolfo (2001), "Decrecimiento y convergencia de los estados mexicanos. Un análisis de panel”, El Trimestre Económico, LXVIII, (272), Fondo de Cultura Económica, México, pp. 603-629.

Cermeño, Rodolfo, David Mayer-Foulkes y Ariadna Martínez-González (2009), "Convergencia, divergencia y estratificación. Estudio comparativo de la dinámica de crecimiento de la manufactura en los municipios mexicanos y los condados estadunidenses", $E l$ Trimestre Económico, LXXVI (2) (302), Fondo de Cultura Económica, México, pp. 349-378.

Chiquiar, Daniel (2005), "Why Mexico's regional income convergence broke dow", Journal of Development Economies, 77 (1), Elsevier, pp. 257-275.

Dallerba, Sandy (2005), "Distribution of regional income and regional funds in Europe 1989-1999: An exploratory spatial data analysis", The Annals of Regional Science, 39 (1), Springer Berlin Heidelberg, pp. 121-148.

Díaz-Bautista, Alejandro (2003), "Apertura comercial y crecimiento regional", Comercio Exterior, 53 (11), Banco Nacional de Comercio Exterior, México, pp. 995-1000.

Díaz-Pedroza, Jesús, Armando Sánchez-Vargas y Miguel Ángel MendozaGonzález (2009), "Convergencia hacia la economía regional líder en México. Un análisis de cointegración en panel”, El Trimestre 
Económico, LXXVI (2), Fondo de Cultura Económica, México, pp. 407-431.

Esquivel, Gerardo (1999), "Convergencia regional en México, 1940 1995”, El Trimestre Económico, 264 (66), Fondo de Cultura Económica, México, pp. 725-761.

Esquivel, Gerardo y Miguel Messmacher (2002), "Sources of regional (non) convergence in Mexico", documento de trabajo, El Colegio de México, México.

Evans, Paul y Georgios Karras (1996), "Convergence revisited", Journal of Monetary Economics, 37 (2-3), Elsevier, pp. 249-65.

Fujita, M., P. Krugman y A. J. Venables (1999), The spatial economy, cities, regions and international trade, The MIT Press, Cambridge.

Galindo, M. A. y G. Malgesini (1994), Crecimiento económico, Editorial McGraw-Hill, Nueva York.

González-Rivas, Marcela (2007), "The effects of trade openness on regional inequality in Mexico", The Annals of Regional Science, 41 (3), Springer International Publishing, pp. 545-561.

Hansen, Bruce (1999), "Testing for linearity”, Journal of Economic Surveys, 13 (5), Wiley Blackwell, pp. 551-576.

Im, K., H. Pesaran y Y. Shin (1997), "Testing for unit roots in heterogeneous panels", mimeo, Trinity College, University of Cambridge, Cambridge.

Islam, Nazrul (1995), "Growth empirics: a panel data approach", The Quarterly Journal of Economics, 110 (4), Jstor, pp. 1127-1170.

Islam, Nazrul (1998), "Convergence: variation in concept and empirical results", Department of Economics Emory University, Emory College of Arts and Sciences, Atlanta, pp. 1-60.

Juan-Ramón, Hugo y Luis Rivera-Batiz (1996), "Regional growth in Mexico, 1970-1993”, working paper, núm. 96/92, International Monetary Fund, Washington, pp. 1-36. 
Kaldor, Nicholas (1970), "The case for regional policies", Scottish Journal of Political Economy, 17 (3), Wiley Online Library, pp. 337-348.

Kaldor, Nicholas (1981), "The role of increasing returns, technical progress and cumulative causation in the theory of international trade and economic growth", Economie Appliquée: archives de l'Institut de Sciences Mathématiques et Economiques Appliquées, 34 (4), Journal of Economic Analysis, París, pp. 593-617.

Koopmans, Tjalling (1965), "On the concept of optimal economic growth", The Econometric Approach to Development Planning, vol. 28, Pontificiae Academiae Scientiarum scripta varia, Vatican City, pp. 225-300.

Larsson, Rolf, Johan Lyhagen y Mickael Löthgren (2001), "Likelihood based cointegration tests in heterogeneous panels", The Econometrics Journal, 4 (1), Wiley, pp 109-142.

Levin, Andrew y Chien-Fu Lin (1993), "Unit root tests in panel data: new results", discussion paper 93-56, University of California, San Diego.

Levin, Andrew y Chien-Fu Lin (2001), "Unit root tests in panel data: asymptotic and finite-sample properties", discussion paper, Federal Reserve Board of Governors and National Taiwan University, Taipéi.

Mankiw, N. Gregory, David Romer y David N. Weil (1992), "A contribution to the empirics of economic growth", Quarterly Journal of Economics, núm. 107, The National Bureau of Economic Search, Cambridge, pp. 407-437.

Mark, Nelson y Donggyu Sul (2003), "Cointegration vector estimation by panel dols and long-run money demand", Bulletin of Economics and Statistics, 65 (5), University of Oxford, Oxford, pp. 655-680.

Mayer-Foulkes, David (2005), "Human development traps and economic growth”, en Guillem López-Casanovas, Berta Rivera y Luis Currais (eds.), Health and economic growth: Findings and policy implications, MIT Press, Cambridge, pp. 1-26. 
Mayer-Foulkes, David (2009a), "Economic geography of human development: stratified growth in Bolivia, Brazil, Guatemala and Peru", <http://ssrn.com/abstract=1287952>, 25 de enero de 2013.

Mayer-Foulkes, David (2009b), "The cognitive transition in Mexico: economic geography and local governance impacts", <http://ssrn. com/abstract=1303867>, 25 de enero de 2013.

Mayer-Foulkes, David (2010), "Globalization and the human development trap", en Machiko Nissanke y Erik Thorbecke (eds.), The poor under globalization in Africa, Asia, and Latin America, Oxford University Press, Oxford, pp. 39-67.

Mendoza-González, Miguel Ángel (2004), "La dinámica económica regional en México, 1940-2002", Territorio y Economía, núm. 7, Sistema de Información Regional de México, México, pp. 12-22.

Mendoza-González, Miguel Ángel (2012), “Dinámica económica regional de largo plazo en México: 1940-2010”, en Miguel Ángel Mendoza, Luis Quintana-Romero y Normand Asuad (coords.), Análisis espacial y regional: Crecimiento, concentración económica, desarrollo y espacio, Plaza y Valdés-unam, México, pp. 21-43.

Mendoza-González, Miguel Ángel (2014), “Metodología de interpolación estructural-espacial para la generación de una serie continua del PIB y población por entidad federativa, 1970-2012", documento de trabajo, Facultad de Economía-unam, México.

Mora, Toni (2005), "Evidencing european regional convergence clubs with optimal grouping criteria", Applied Economics Letters, 12 (15), Taylor \& Francis Online, pp. 937-948.

Myrdal, Gunnar (1957), Economic theory and underdeveloped regions, Duckworth, Londres.

Ottaviano, Gianmarco y Jacques-Francois Thisse (2004), "Agglomeration and economic geography”, en J.V. Henderson y J. F. Thisse (eds.), Handbook of Regional and Urban Economics, vol. 4, Elsevier, pp. 1-44. 
Pedroni, Peter (1999), "Critical values for cointegration tests in heterogeneous panels with multiple regressors", Oxford Bulletin of Economics and Statistics, vol. 61, Blackwell Publishers, pp. 653-678.

Perroux, François (1950), "Economic space: theory and application", Quarterly Journal of Economics, 64 (1), Oxford Journals, pp. 89-104.

Perroux, François (1955), "Note sur la notion de pole de croissance", Economie Appliquée, núm. 8, Institut de Sciences Mathématiques et Economiques Appliquées, París, pp. 161-171.

Petrakos, Rodriguez-Pose y Rovolis (2005), "Growth, integration, and regional disparities in the European", Environment and Planning, 37 (10), Sage Journals, pp. 1837-1855.

Ramsey, Frank Plumpton (1928), "A mathematical theory of savings", The Economic Journal, 38 (52), Royal Economic Society, Jstor, pp. 543-559.

Rodríguez, Andrés y Javier Sánchez (2002), “The impact of trade liberalization on regional disparities in Mexico", Growth and Change, 33 (1), Wiley Online Library, pp. 72-90.

Rodríguez-Oreggia, Eduardo (2002), "Polarization of income under structural changes: winners and losers of regional growth in Mexico", working paper, pp. 4-27.

Rodríguez-Oreggia, Eduardo (2005), "Regional disparities and determinants of growth in Mexico", The Annals of Regional Science, 39 (2), Springer, pp. 207-220.

Sala-i-Martin, X. (1996), "The classical approach to convergence analysis", The Economics Journal, 106 (437), Royal Economic Society, pp. 1019-1036.

Solow, Robert-Merton (1956), "A contribution to the theory of economic growth", The Quarterly Journal of Economics, 70 (1), The MIT Press, pp. 65-94.

Tong, Howell (1978), "On a threshold model”, en C. Chen (ed.), Pattern recognition and signal processing, Sijhoff and Noordhoff, Amsterdam, pp. 575-586. 
Recibido: 5 de abril de 2014. Reenviado: 19 de noviembre de 2014. Aceptado: 24 de noviembre de 2014.

Domingo Rodríguez-Benavides. Es doctor en economía por la Universidad Nacional Autónoma de México, maestro en economía y maestro en finanzas por la misma institución. Es profesor-investigador titular de tiempo completo en la Escuela Superior de Economía del Instituto Politécnico Nacional. Es miembro del SNI, nivel I. Tiene el perfil PROMEP otorgado por la SEP. Entre sus artículos recientes destacan, en coautoría: "La hipótesis de convergencia en América Latina: Un análisis de cointegración en panel”, EconoQuantum, 9 (2), Universidad de Guadalajara, Jalisco, pp. 99-122 (2012); "La ley de Wagner versus la hipótesis keynesiana: el caso de México, 1950-2009”, Investigación Económica, LXXII (283), unam, México, pp. 69-98 (2013); "Desarrollo económico y gasto público de las entidades federativas en México: Análisis de cointegración en panel y la ley de Wagner", Gestión y Política Pública, XXIII (2), Centro de Investigación y Docencia Económicas, A. C., México, pp. 299-330 (2014).

Miguel Ángel Mendoza-González. Es economista, maestro en ciencias económicas y doctor en economía por la UNAM. Fue coordinador de Campo de Conocimiento de Economía Urbana y Regional de la Facultad de Economía de la unam, del Seminario del doctorado del Campo de Conocimiento en Economía Urbana y Regional, y representante del rector en la Junta de Gobierno del Colegio del Estado de Hidalgo. Profesor titular "C" de tiempo completo definitivo, con una antigüedad docente de 24 ańos; tutor de maestría y de doctorado en el posgrado de la Facultad de Economía de la UnAm. Es coautor de los libros: Eudoxio: Un modelo macroeconométrico para la economía mexicana, UNAM, México (2000); Tópicos de economía matemática y econometría, unaM, México (1998); Econometría básica, modelos y aplicaciones a la economía mexicana, FEs Acatlán-DGapa-Plaza y Valdés, México (2008); Análisis espacial y regional: crecimiento, concentración económica, desarrollo y espacio (2012), unam-Plaza y Valdés, México, 371 pp. (2012). Tiene hasta el momento 40 artículos de divulgación, 20 artículos arbitrados publicados y 16 capítulos de libros. Sus trabajos más recientes son, en coautoría: "Regional output growth and the impact of macroeconomic shocks in Mexico", International Review of Applied Economics, 28 (3) International review of applied economics, Abingdon, Routledge, pp. 293-310 (2014); "Women's industrial employment in Mexico, measures of discrimination and segregation", Journal of Business and Economics, 3 (6), Air University, Pakistán, pp. 410-423 (2012); "Human capital and growth in Latin America", en 
Juan R. Cuadrado-Roura y Patricio Aroca (eds.), Regional Problems and Policies in Latin America, Springer-Verlag Publics, pp. 359-377 (2013); "Externalidades de capital humano y espaciales, su influencia en el crecimiento económico de las ciudades de México", en Marcos Valdivia López y Javier Delgadillo Macías (coords.), La geografía y la economía en sus vinculos actuales. Una antología comentada del debate contemporáneo, CRIMIIEc-unam, México, pp. 221-232 (2013); "El debate teórico sobre la convergencia o divergencia económica regional”, en Ignacio Perrotini Hernández (ed.), Politica Económica: análisis monetario, regional e institucional, Benemérita Universidad Autónoma de Puebla, Puebla, pp. 157173 (2013).

Francisco Venegas-Martínez. Tiene postdoctorado en finanzas en Oxford University, doctorado en matemáticas en Washington State University y doctorado en economía en Washington State University. Es profesor-investigador titular " $\mathrm{C}$ " de tiempo completo definitivo de la Escuela Superior de Economía del Instituto Politécnico Nacional. Es miembro del sNi, nivel III. Fue ganador de la Presea "Lázaro Cárdenas", 2012, la más alta distinción que otorga el IPN a un profesor-investigador. Ganador del Premio a la Investigación Aplicada en el IPN, en 2011. Sus artículos recientes, en coautoría: "Inflation and private sector bank credit in Mexico: an ARDL-Bounds testing approach", Latin American Economic Review, en prensa; "Efectos del gasto en seguridad pública en el crecimiento económico: un modelo macroeconómico estocástico", Investigación Económica, 73 (288), unam, México, pp. 117-147 (2014); "Valuación de opciones europeas sobre AMX-L, WALMEX-V y GMEXICO-B: calibración de parámetros de volatilidad estocástica con funciones cuadráticas de pérdida", El Trimestre Económico, 81 (324), Fondo de Cultura Económica, México, pp. 943-988 (2014). 